\title{
Central pontine myelinolysis associated with hypokalaemia in anorexia nervosa
}

\author{
T Sugimoto, T Murata, M Omori, Y Wada
}

J Neurol Neurosurg Psychiatry 2003;74:353-355

A 31 year old man was admitted to hospital with of anorexia, binge eating, and self induced vomiting. On admission, he showed a pronounced low weight and disturbance of the body image and was diagnosed as having anorexia nervosa. In addition, electrolyte abnormalities, mainly hypokalaemia, and increased serum renin and aldosterone concentrations were recorded, suggesting pseudo-Bartter syndrome as a complication. Under frequent monitoring of the serum potassium and sodium concentrations, serum electrolytes were gradually corrected, but brain magnetic resonance imaging revealed reversible central pontine myelinolysis (CPM). Although attention has been mainly paid to the association of CPM with rapid correction of hyponatraemia and abnormal osmolality, this case report strongly suggested the involvement of hypokalaemia in the pathogenesis of CPM.

\footnotetext{
C
} entral pontine myelinolysis (CPM) is a demyelinative lesion in the central pons that was first reported in necropsy cases of alcoholism and malnutrition. ${ }^{1}$ CPM is characterised by disturbance of consciousness, quadriparesis, and mutism and has been considered to have a poor prognosis. ${ }^{2}$ However, with advances in diagnostic imaging techniques such as magnetic resonance imaging (MRI), some patients with asymptomatic CPM, which had been overlooked in the past, have been reported. ${ }^{34}$ Since the pathogenesis of CPM, abnormalities in serum electrolytes and serum osmolality have mainly attracted attention, and many patients with CPM caused by rapid correction of hyponatraemia have been reported..$^{5}$ However, normonatraemic and hypernatraemic patients have also been described. ${ }^{7-9}$ Recently, hypokalaemia related CPM irrespective of the serum sodium concentration has been also reported. ${ }^{10}{ }^{11}$ Thus, there is no consensus about what abnormalities in electrolytes or osmolality are important pathogenic factors of CPM.

Several patients with CPM as a complication of anorexia nervosa have been reported. In each patient, the mechanism of the development of CPM was discussed in association with serum electrolyte abnormalities attributable to malnutrition, excessive water intake, or laxative and/or diuretic misuse and their rapid correction. ${ }^{12}{ }^{13}$ We report a patient with anorexia nervosa complicated by pseudo-Bartter syndrome who repeated self induced vomiting and showed a reversible CPM lesion on MRI images despite gradual correction of electrolyte abnormalities mainly hypokalaemia.

\section{CASE REPORT}

The patient was a 31 year old man without remarkable family history or past history. Though his school record was good, he developed pilonidal abscess in the gluteal region at the age of 14 years and could not concentrate on studying because of pain induced stress and repeated operations. He could not enter the high school he hoped and began to repeatedly exhibit anorexia, binge eating, and self induced vomiting. The body weight was $84 \mathrm{~kg}$ at the age of 14 years but decreased to $50 \mathrm{~kg}$ at the age of 25 years when he got a job. After he began to work on a day/night shift in April 2001, the frequency of binge eating and self induced vomiting increased. On 23 July, he visited a local hospital because of general malaise. At this time, the body weight was $34 \mathrm{~kg}$ (body mass index $=12.7$ ), and abnormalities of electrolytes mainly hypokalaemia were observed (serum sodium concentration, $140 \mathrm{mEq} / \mathrm{l}$; serum potassium concentration, $2.8 \mathrm{mEq} / \mathrm{l}$ ). He was referred to the medical department of our hospital and admitted on the same day. Immediately, intravenous fluid therapy via a peripheral vein was initiated for the normalisation of the hypokalaemia and nutritional supplementation. Based on increased serum renin and aldosterone values and hypokalaemic alkalosis (renin, $24.6 \mathrm{ng} / \mathrm{ml} / \mathrm{h}$; aldosterone, $572 \mathrm{ng} / \mathrm{dl} ; \mathrm{pH}, 7.51$ ), a diagnosis of pseudo-Bartter syndrome caused by self induced vomiting was made. After admission, self induced vomiting was observed after almost each meal, and the body weight did not increase. On 20 August, intravenous hyperalimentation (IVH) was started, but behavioural problems such as going out without permission and binge eating appeared. On 6 September, he was transferred to the psychiatric ward. During this period, the serum potassium concentration markedly changed, often resulting in severe hypokalaemia (fig 1). However, the serum sodium concentration and osmolality changed almost in the normal range (fig l).

At the time of the transfer to the psychiatric ward, marked low body weight ( $<60 \%$ of standard weight) was observed. He had a strong fear of obesity and misperception of the body image and was diagnosed as having anorexia nervosa according to the DSM-IV diagnostic criteria. ${ }^{14}$ On 10 September, brain MRI revealed hyperintensity in the central pons on T2 weighted images (fig 2A), indicating asymptomatic CPM (absence of neurological or psychiatric symptoms in the foreground). IVH was continued and changed to oral ingestion when the body weight reached $40 \mathrm{~kg}$. To inhibit self induced vomiting, behavioural restrictions were imposed (stepwise according to body weight) by making him to stay at rest in his room for a certain time after each meal. However, he could not heed these behavioural restrictions and repeatedly vomited in secret, and the body weight decreased to $32 \mathrm{~kg}$. Therefore, from 15 November, IVH was performed again, and behavioural restrictions were tightened. The frequency of vomiting decreased, and abnormalities of electrolytes, mainly hypokalaemia, were normalised. Subsequently, CPM findings on MRI images disappeared ( fig 2B).

Abbreviations: CPM, central pontine myelinolysis; MRI, magnetic resonance imaging 


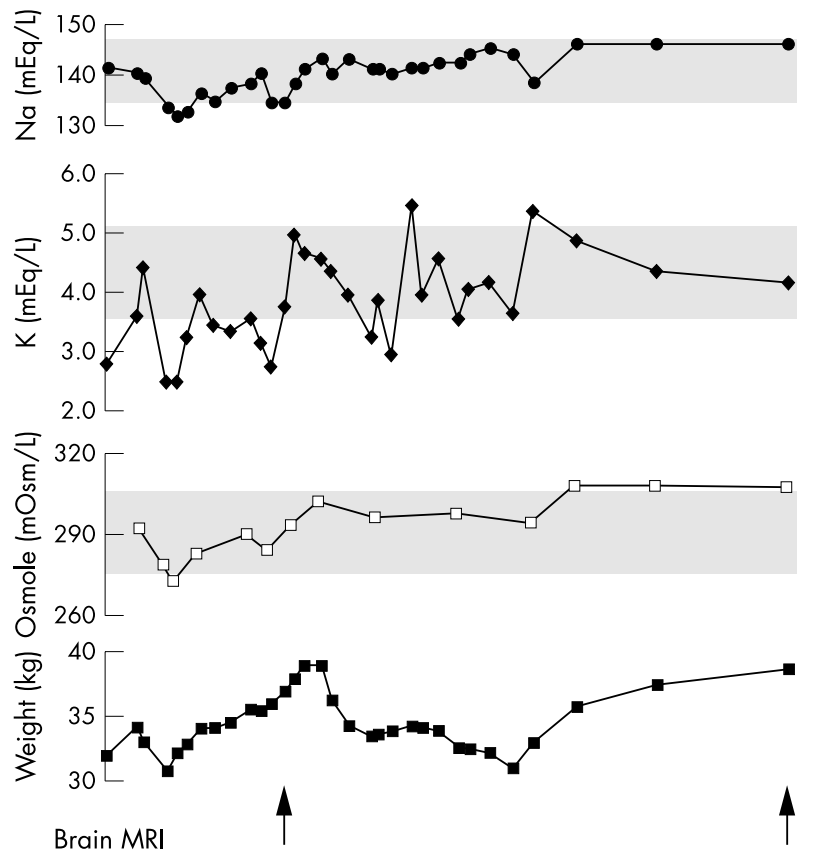

Brain MRI

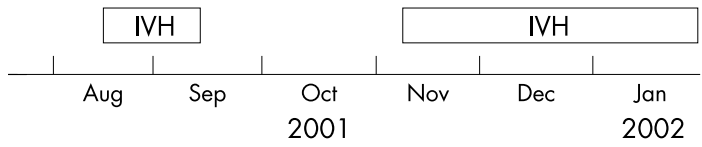

Figure 1 Clinical course after admission. Arrows indicate MRI examinations. Dotted areas indicate normal ranges of serum electrolytes or osmolality. IVH, intravenous hyperalimentation.

\section{DISCUSSION}

CPM produces neurological symptoms such as quadriparesis, pseudobulbar palsy, oculomotor paresis, and locked in syndrome $^{12}$ and psychiatric symptoms such as changes in the level of consciousness and behaviour or cognitive dysfunction. ${ }^{13}{ }^{15}{ }^{16}$ In this patient, none of these clinical symptoms was observed in the foreground, and asymptomatic CPM was considered. With recent advances in diagnostic imaging techniques such as MRI, cases of asymptomatic CPM, which may have been overlooked previously, have been reported. ${ }^{34}$ There may be many patients with latent reversible CPM as in this patient.

The pathogenic factors of CPM first reported were alcoholism, malnutrition, and chronic liver disease. ${ }^{12}$ Subsequently, rapid correction of hyponatraemia has attracted attention as an important factor. ${ }^{5617}$ In addition, abnormal osmotic pressure $^{111_{18}^{18}}$ and electrolyte abnormalities mainly hypokalaemia ${ }^{1019}$ and acute changes in the blood glucose concentration $^{20}$ have been also suggested. In this patient with anorexia nervosa, there was no history of alcohol misuse or liver dysfunction, and the serum sodium concentration and osmole changed almost in the normal range. The fasting blood glucose concentration was $70-100 \mathrm{mg} / \mathrm{dl}$ without marked changes. On the other hand, the serum potassium concentration considerably changed, often showing severe hypokalaemia. Patients who vomit lose hydrogen, chloride, and water and develop hypokalaemia not only because of shift of potassium ions into cells as a result of alkalosis, but also because of increased potassium ion excretion in the urine as a result of reabsorption of hydrogen ions. ${ }^{21}$ Secondary aldosteronism attributable to the decrease of total body water also causes an increase of potassium ion excretion in the urine. ${ }^{22}$ As this patient also frequently repeated vomiting, pseudo-Bartter syndrome may have developed by such a mechanism, resulting in marked hypokalaemia. Our review of the literature showed many patients with CPM complicated by hypokalaemia but only three patients with CPM with hypokalaemia but normonatraemia ${ }^{10}{ }^{19}$ as was observed in our patient. The underlying disease was chronic alcohol misuse ${ }^{10}$ in two of the three patients and Sjögren's syndrome ${ }^{19}$ in the other; anorexia nervosa was not observed.

The developmental mechanism of CPM is unknown. Proposed hypotheses include osmotic injury to the endothelium resulting in release of myelinotoxic factors or vasogenic oedema ${ }^{17}$ and brain dehydration resulting in separation of the axon from its myelin sheath with resultant injury of oligodendrocytes, particularly at interfaces of grey and white matter. ${ }^{23}$ However, a recent study suggested that CPM tends to occur in hyponatraemia complicated by hypokalaemia because a decreased concentration of $\mathrm{Na}$, K-ATPase in endothelial cell membrane during hypokalaemia may predispose the cell susceptible to injury by osmotic stress associated with the rapid rise in the serum sodium concentration. ${ }^{11}$ This patient also developed hypokalaemia caused by frequent self induced vomiting. Therefore, there is a possibility that CPM was induced by a slight increase in osmotic pressure attributable to fluid infusion such as of electrolytes and glucose in the presence of severe hypokalaemia (even if the increase rate was so low as not to induce injury in the normal state). ${ }^{64}$

To our knowledge, there have been reports of only two patients with anorexia nervosa in whom CPM developed after rapid correction of marked serum electrolyte abnormalities attributable to water intoxication (excessive water intake) or
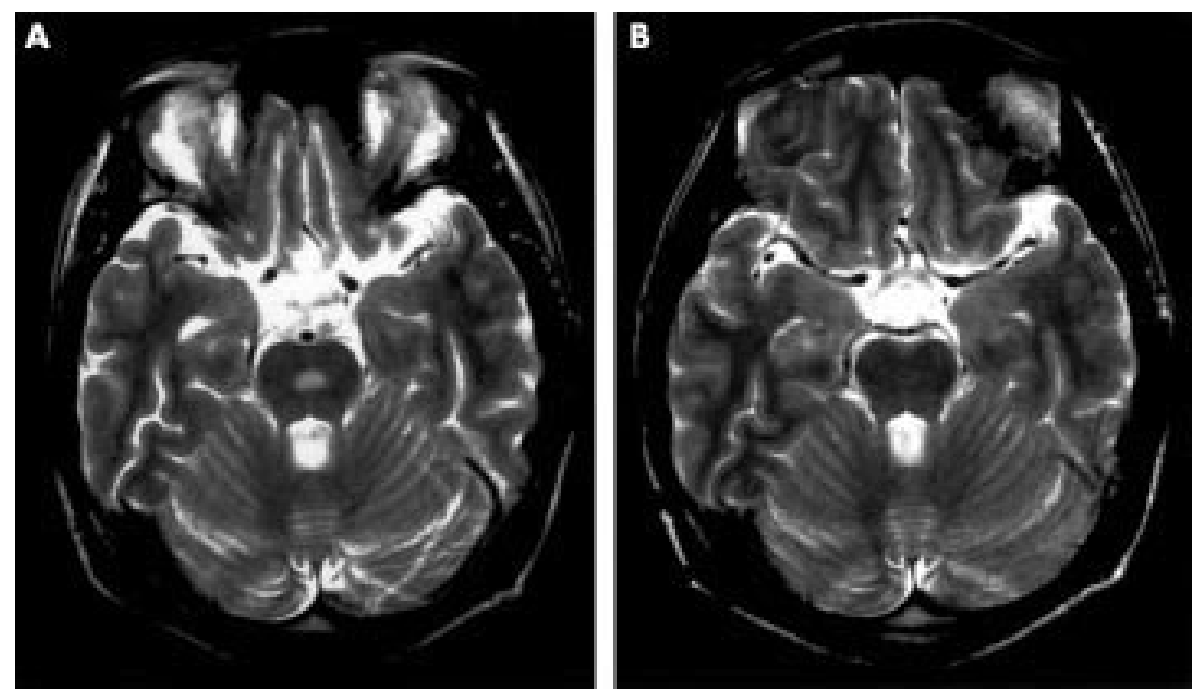

Figure 2 Serial axial T2 weighted MR images (TR=3200 ms, TE=82.9 $\mathrm{ms}$ ) focusing on the brain stem. (A) MRI about two months after admission showed a central pontine hyperintense lesion. (B) A completely normal brain stem was found about a half year after admission. 
laxative misuse. ${ }^{12}{ }^{13}$ In our patient, however, neither excessive water intake nor laxative abuse was observed, and electrolyte abnormalities, mainly hypokalaemia, caused by frequent self induced vomiting developed (the serum sodium concentration was nearly normal), and CPM occurred despite gradual electrolyte correction. In patients with anorexia nervosa, the complication rate of vomiting is $28 \%$ and that of hypokalaemia is $20 \% .{ }^{21}$ This case report suggests that further caution is necessary when serum electrolytes are corrected in such patients.

\section{Authors' affiliations}

T Sugimoto, T Murata, M Omori, Y Wada, Department of

Neuropsychiatry, Fukui Medical University, Fukui, Japan

Competing interests: none declared.

Correspondence to: Dr T Murata, Department of Neuropsychiatry, Fukui Medical University, Matsuoka-cho, Fukui 910-1193, Japan; tmurata@fmsrsa.fukui-med.ac.jp

Received 9 July 2002

Accepted in revised form 10 November 2002

\section{REFERENCES}

1 Adams RD, Victor M, Mancall EL. Central pontine myelinolysis: a hitherto undescribed disease occurring in alcoholic and malnourished patients. Arch Neurol Psychiatry 1959;81:154-72.

2 Messert B, Orrison WW, Hawkins M, et al. Central pontine myelinolysis: considerations on etiology, diagnosis, and treatment Neurology 1979;29: 147-60.

3 Pfister HW, Einhäupl KM, Brandt T. Mild central pontine myelinolysis: a frequently undetected syndrome. Eur Arch Psychiatr Neurol Sci 1985;235:134-9.

4 Girmenia F, Colosimo C, Di Biasi C, et al. Central pontine myelinolysis: report of an asymptomatic case. Ann ltal Med Int 1995;10:53-4.

5 Laureno $\mathbf{R}$. Central pontine myelinolysis following rapid correction of hyponatremia. Ann Neurol 1983;13:232-42.

6 Sterns RH, Riggs JE, Schochet SS Jr. Osmotic demyelination syndrome following correction of hyponatremia. N Engl J Med 1986;314:153542.
7 DeWitt LD, Buonanno FS, Kistler JP, et al. Central pontine myelinolysis: demonstration by nuclear magnetic resonance. Neurology 1984;34:570-6

8 Brunner JE, Redmond JM, Haggar AM, et al. Central pontine myelinolysis after rapid correction of hyponatremia: a magnetic resonance imaging study. Ann Neurol 1988;23:389-91.

9 Mast H, Gordon PH, Mohr JP, et al. Central pontine myelinolysis: clinical syndrome with normal serum sodium. Eur J Med Res 1995;1:168-70.

10 Bähr M, Sommer N, Petersen D, et al. Central pontine myelinolysis associated with low potassium levels in alcoholism. I Neurol 1990;237:275-6.

11 Lohr JW. Osmotic demyelination syndrome following correction of hyponatremia: association with hypokalemia. Am J Med 1994;96:408-13

12 Greenberg WM, Shab PJ, Vakharia M. Anorexia nervosa/bulimia and central pontine myelinolysis. Gen Hosp Psychiatry 1992;14:357-8.

13 Amann B, Schäfer $M$, Sterr A, et al. Central pontine myelinolysis in a patient with anorexia nervosa. Int J Eat Disord 2001;30:462-6.

14 American Psychiatric Association. Diagnostic and statistical manual of mental disorders. 4th edn. Washington, DC: APA, 1994.

15 Silbert PL, Knezevic WV, Peake HI, et al. Behavioural changes due to pontine and extrapontine myelinolysis. Med J Aust 1992;157:487-8.

16 Vermetten E, Rutten SJE, Boon PJ, et al. Neuropsychiatric and neuropsychological manifestations of central pontine myelinolysis. Gen Hosp Psychiatry 1999;21:296-302.

17 Norenberg MD. A hypothesis of osmotic endothelial injury: a pathogenic mechanism in central pontine myelinolysis. Arch Neurol 1983:40:66-9

18 Mckee AC, Winkelman MD, Banker BQ. Central pontine myelinolysis in severely burned patients: relationship to serum hyperosmolality. Neurology 1988;38:1211-17.

19 Nagashima K, Wakayama M, Yaguchi M, et al. A patient with Sjögren syndrome with central pontine myelinolysis and hypokalemic myopathy. Rinsho Shinkeigaku 1996;36:1240-4.

20 Esforzado N, Poch E, Cases A, et al. Central pontine myelinolysis secondary to frequent and rapid shifts in plasma glucose in a diabetic haemodialysis patient. Nephrol Dial Transplant 1993:8:644-6.

21 Koh E, Onishi T, Morimoto S, et al. Clinical evaluation of hypokalemia in anorexia nervosa. Jpn J Med 1989;28:692-6.

22 Warren SE, Steinberg SM. Acid-base and electrolyte disturbances in anorexia nervosa. Am J Psychiatry 1979;136:415-18.

23 Sterns RH. The management of symptomatic hyponatremia. Semin Nephrol 1990;10:503-14.

24 Ayus JC, Krothapalli RK, Arieff Al. Treatment of symptomatic hyponatremia and its relation to brain damage. N Engl J Med 1987;317:1190-5.

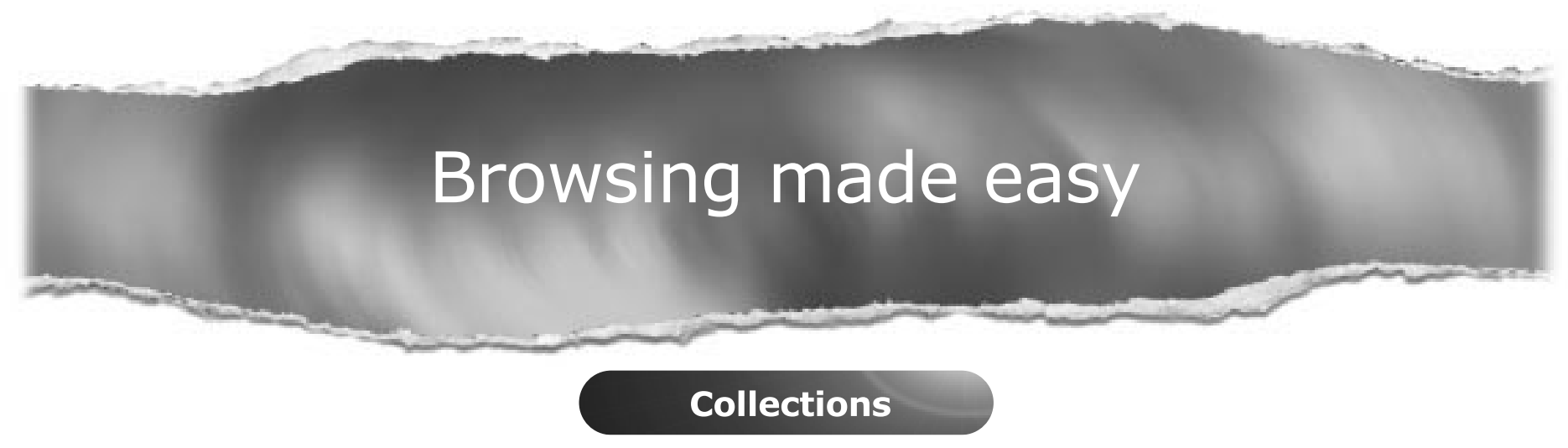

With a single click Collections allows you to find all articles that have been published in your chosen subject. Select from over 200 clinical and non-clinical topic collections and/or cross search other specialist journals, the BMJ and Cochrane Reviews

www.jnnp.com 